IRA-International Journal of Technology \& Engineering ISSN 2455-4480

Proceedings of the

International Conference on Science \& Engineering for Sustainable Development (2017)

Pg. no. $122-136$

Published by: Institute of Research Advances https://research-advances.org/index.php/IRAJTE

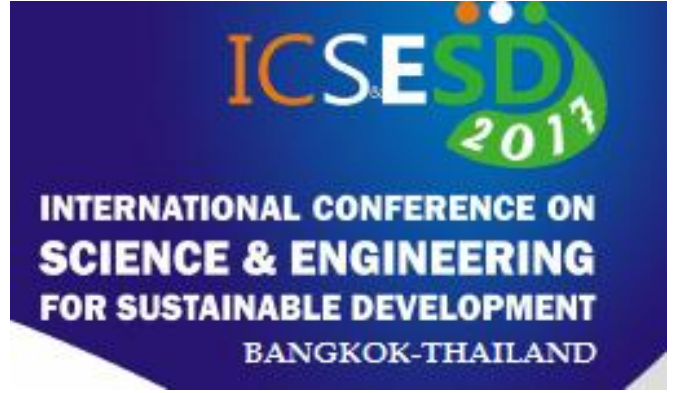

\title{
Performance Analysis Methods of Non- directed IR Wireless Channel Preparation of Papers for Short Range Inside the Building
}

Prakash H. Patil

D. Y. Patil College of Engineering, Ambi Pune,(MS) India

Type of Review: Originality Check \& Peer Review under the responsibility of the Scientific Committee of the Conference and The Institution of Engineers (India). DOI: http://dx.doi.org/10.21013/jte.ICSESD201713

How to cite this paper:

Patil, P. (2017). Performance Analysis Methods of Non-directed IR Wireless Channel Preparation of Papers for Short Range Inside the Building. Proceedings of the International Conference on Science \& Engineering for Sustainable Development (2017), 122-136. doi: http://dx.doi.org/10.21013/ite.ICSESD201713

(C) International Conference on Science \& Engineering for Sustainable Development\& The Institution of Engineers (India).

\section{(cc) Br-No}

This work is licensed under a Creative Commons Attribution-Non Commercial 4.0 International License subject to proper citation to the publication source of the work.

Disclaimer: The conference papers as published by the Institute of Research Advances (IRA) are the views and opinions of their respective authors and are not the views or opinions of the IRA. The IRA disclaims of any harm or loss caused due to the published content to any party. 


\section{ABSTRACT}

Nowadays due to the tremendous growth of residential and commercial buildings it is very much essential to design and simulate IR Wireless channel inside the building. Here we need to consider the room configurations and several techniques such as FFT and wavelet analysis for the estimation of received power signal strength. Also, it is required to ensure high speed, short range and secured optical wireless communication inside the building by considering different obstacles and using IR transmitter and receiver for the improved BER.

Keywords: IR Wireless Channel, Short Range, FFT, Wavelet.

\section{Introduction}

The infrared communication system is confined within a specific infrared room. Every room is considered as one cell and many adjacent rooms act as neighboring cells. The bandwidth used in one room can be reused in other rooms and it is compatible worldwide. The only noise present in indoor infrared communication is the ambient light. The ambient light is formed by sunlight, incandescent lamps and fluorescent lamps. Infrared links cannot continue operations in direct sunlight, unless spatial ial sources of ambient light cause a periodic interference sign filters or very directional sources and receivers are used. Artificial in IR wireless receivers. If this interference is disregarded, it may degrade the performance of IR link. The performance degradation of the IR link arises due to wide spectrum and emission of high power IR radiation. [1] [2] [3].

Even though the infrared channel is related to the linear Gaussian channel, there is a distinction among the two. The input of infrared channel is represented by powerfully while that of radio channel is represented by amplitude. Also, the signal has to be positive and its average value should not exceed a set value for safety purpose [4].IR radiation does not penetrate opaque objects, such as walls, partitions etc. and thus provides highly secured communication since the radiation is confined within the cell. Alternatively, an infrared link offers minimum coverage. Also, base stations should be positioned within every room, where IR communication is in use. Earlier researchers have modeled indoor reflections of infrared with the purpose of determining the distribution of power throughout a room. Gfeller and Bapst presented similar analysis in [5] which accounts for single reflections only; Hash et al. extended their procedure to consider double reflections as well [6]. The simulations in these works were meant for a link budget analysis; thus, only the total power reaching the receiver was estimated. In other words, they were concerned only with the time integral of $\mathrm{h}(\mathrm{t})$. Since power budgets typically have built-in safety margins, the accuracy provided by considering only first- and second-order reflections was sufficient. Hortensius extended the Gfeller and Bapst model to calculate an impulse response, accounting for single reflections only [7]. In contrast to prior work, the method described here can compute the impulse response accounting for any number of reflections. This allows accurate power distribution analysis, and, perhaps more importantly, accurate impulse-response analysis. The latter is necessary because signal energy undergoing two or more reflections, although have reduced amplitude, arrives at the receiver much later than first-order reflections. This temporal spread is critical in high-speed applications, in which case higher-order reflections cannot be ignored. 
The basic subsystem of an optical wireless system is transmitted based on Light emitting diodes or Laser diodes (LED's or LDs), the channel (the medium between the transmitter and the receiver) and the receiver based on (PIN or APD based). The link length can vary from a few meters to a few km. Here, we consider the Ceiling Bounce Model as the reference. The intensity modulation with direct detection (IM/DD) is the most practical method deployed for the design analysis of the Nondirected IR wireless communication in indoor environment. In the next section, we define the design constraints of source and reflector models upon which our procedure is based. Section III describes multiple path impulse response. and algorithm. In Section IV we have reported modeling of Non directed IR Wireless channel. The percentage change in bit error rate is presented in Section V. It explained a computer implementation for room configuration and the discussion on simulation results obtained in Section VI The Conclusion is stated in Section VII

\section{Design Analysis of Non Directed IR Wireless Communication System}

The fundamental design constraints of Nondirected Infrared Wireless communication System in indoor environment were described by J. R. Barry [9] Here, the empty rectangular room is taken into account for the simulation of Nondirected IR wireless communication system. The modeling of the transmitter, reflectors, and receiver is described below

\section{A. Transmitter and Receiver Model}

A wide-beam optical source can be represented by position vector $\mathrm{S}$, a unit-length orientation vector, the power $P_{S}$, and a radiation intensity pattern $R \square \square \square \square \square \square$ defined as the optical power per unit solid angle emitted from the source at position $\square \square \square \square \square \square \square$ Following Gfeller [5], transmitter is modeled using a generalized Lambertian radiation pattern.

generalized Lambertian radiation pattern.

$R(\phi)=\frac{(n+1)}{(2 \pi)} P_{\mathrm{S}} \cos ^{n}(\phi) \quad$ For $\phi \in$

Here, $n$ is the mode number of the radiation lobe, which decides the directionality of the transmitter. The transmitters with higher directionality are seen to have greater mode numbers. The coefficient $(n+1) / \square \square \square$ ensures that integrating( $\square)$ over the surface of a hemisphere results in the source power $\mathrm{P}_{\mathrm{S}}$ The radiation mode of $n=1$ corresponds to a traditional Lambertian source. To simplify notation, a point source Tthat emits a unit impulse of optical intensity at time zero is represented by S.

$$
S=\left(\boldsymbol{r}_{S}, \hat{n}_{S}, n\right)^{(2)}
$$

Where $\boldsymbol{r}_{s}$ is its position, $\hat{n_{s}}$ is its orientation, and $n$ is its mode number. Linearity allows us to consider only unit-impulse sources and scale the results for other sources. Similarly, a receiving element $R$ with position $r_{R}$ orientation $n_{r}$, area $A_{R}$ and field of view $F O V$ will be denoted by $R$ 


$$
\mathbb{R}=\left(\boldsymbol{r}_{R}, \hat{\boldsymbol{n}}_{R}, n \text { FOV }\right)
$$

The scalar angle $F O V$ is defined such that a receiver only detects light whose angle of incidence (with respect to the detector normal) is less than FOV. A limited field of view may be an inadvertent effect of detector packaging, or it may be used intentionally to reduce unwanted reflections or noise.

\section{B. Reflection Model.}

Although true reflections contain both specular and diffusive components, we make the simplifying assumption that all reflectors are purely diffusive, ideal Lambertian. Experimental measurements have shown that many typical materials such as plaster walls, acoustic-tiled walls, carpets, and unvarnished wood are well approximated as Lambertian

[8]. The radiation intensity pattern $R(\square)$ emitted by a differential element of an ideal diffuse reflector is independent of the angle of the incident light. This fact is key to our results because it allows us to decompose a reflection into two sequential steps: to model the reflection from a differential reflecting element with area, $\mathrm{dA}$ and reflectivity $\square$, first consider the element as a receiver with area $d A$, and calculate the power $d P$ it receives. Second, model the differential reflector as a source with total power $P$ $d P$ and an ideal Lambertian radiation intensity pattern, as given by (1) with $n=1$.

C. Line-of-Sight (LOS) Impulse Response

If the source $S$ and receiver $R$ are described by (2) and (3) in an indoor environment with no reflectors, if the distance $R$ between transmitter and receiver is large relative to the detector size, so that $R_{\square} \square \wedge A R$, then the received irradiance is approximately constant over the surface of the detector, and furthermore, all of the signal energy will arrive at the receiver at approximately the same time. Thus, using the models described above, the impulse response for this simple system is approximately a scaled and delayed Dirac delta function:

$$
h^{(0)}(t ; S ; R) \approx \frac{(n+1)}{2 \pi} \cos ^{n}(\phi) d \Omega \operatorname{rect}(\theta / F O V) \delta(t-R / c)
$$

Where, $d \square \square$ is the solid angle subtended by the receiver's differentil area (assuming $A_{R^{\ll}} R^{\square}$ )

$$
d \Omega=\cos (\theta) A_{R} / R^{2}(5)
$$

Where, $R$ is the distance between the source and receiver

$$
\mathrm{R}=\left\|r_{S}-r_{R}\right\|(6)
$$

$\square$ is the angle between and $\left.\mathbf{r}_{\mathbf{s}}-\mathbf{r}_{\mathbf{R}}\right)$ :

$\operatorname{COS}(\theta)=\hat{n}_{R}\left(r_{S}-r_{R}\right) / R$

$\operatorname{COS}(\phi)=\hat{n}_{S}\left(r_{S}-r_{R}\right) / R$ 
The rectangular function is defined by:

$\operatorname{rect}(x)=\left\{\begin{array}{l}\{\text { For }|x| \leq 1 \\ 0 \text { For }|x|>1\end{array}\right.$

$C$ is the speed of light. The approximation of (4) approaches equality as the ratio $A_{R} / R$ approaches zero

\section{Multipath Impulse Response}

describe our algorithm for calculating a multiple-path impulse response, after which we discuss a computer implementation.

D. Algorithm

For a given a source Sand receiver Rin a room with reflectors, light from the source can reach the receiver after any number of reflections. Therefore, the impulse response can be written as an infinite sum: [10].

$h \square t \square \square S \square R \square \square=\sum_{k=0}^{\infty} h^{(k)}(t ; S ; R)(7)$

where $h^{\square} \square(t)$ is the response of the light that undergoes exactly $k$ reflections. The line-of-sight response $h \square(t)$ is given by (4), while higher-order reflections $(k>0)$ can be calculated recursively.

$h^{k}(t ; S ; R)=\int_{S} h^{(0)}(t$

风) $\boldsymbol{h}^{(k-1)}$

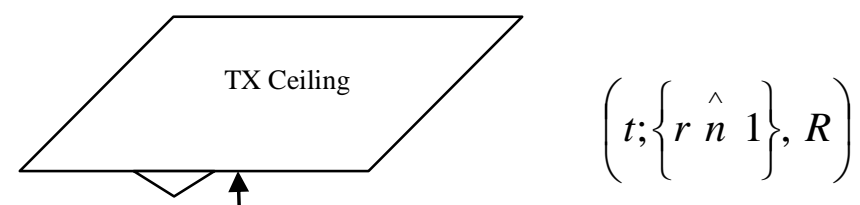


Fig. 1. TX and RX Geometry for modeling of IR channel in FFT Domain

Where the symbol $\square \square$ denotes convolution. More explicitly,

Substituting (4) and performing the convolution results we get,

$h^{k}(t ; S, R)=$

$\frac{n+1}{2 \pi} \sum_{i=1}^{N} \frac{\rho_{i} \cos ^{n}(\phi) \cos (\theta)}{R^{2}} \operatorname{rect}(2 \theta / \pi)$

$B^{(k-1)}(t-R / c ;\{r, n, 1\}, R) \nabla A$

The integrations in (8) and (9) are performed with respect to $\mathbf{r}$ on the surface $S$ of all reflectors. Here, is the normal to the surface $S$ at position $\mathbf{r}, \square_{\mathbf{r}}$ is the reflectivity at position $\mathbf{r}, R \square \square \square \square \mathbf{r}-\mathbf{r}_{S}$

$\operatorname{Cos}(\square) \square \square \mathbf{r}\left(-\mathbf{r}_{S}\right) / R$, and

$$
\cos (\theta)=\hat{n} \cdot\left(r_{S}-r\right) / R
$$

Equation (11) quantifies the main theoretical result. Intuitively, it is further observed that the $k$-bounce impulse response from a single point-source scan be found in two steps: First finding the distribution and timing of the power fromsonto the reflecting walls and second Using the walls as a distributed light source, computing the $(k-1)$-bounce impulse response.

The integral in (11) can be calculated numerically by breaking the reflecting surfaces into numerous small

reflecting elements, each with area $\square A$. Thus, $h^{(k)}(t)$ can be approximated by

$$
\begin{aligned}
& h^{k}(t ; S, R) \approx \sum_{i=1} h^{(0)}\left(t ; S, \varepsilon_{i}\right) \otimes h^{(k-1)}\left(t ; \varepsilon_{i}, R\right) \frac{n+1}{2 \pi} \sum_{i=1}^{N} \frac{\rho_{i} \cos { }^{n}(\phi) \cos (\theta)}{R^{2}} \operatorname{rect}(2 \theta / \pi) \\
& h^{(k-1)}(t-R / c ;\{r, n, 1\}, R) \nabla A
\end{aligned}
$$

TABLE I: SIMULATION PARAMETERS FOR FFT ANALYSIS

\begin{tabular}{|l|c|c|}
\hline \multicolumn{2}{|c|}{ Parameters / Room Configuration } & Values \\
\hline Rom Dimension & Length $(\mathrm{X})$ & 6 \\
(Length)X(Width)X (Height) & Width Y) & 4 \\
& Height $(\mathrm{Z})$ & 3 \\
\hline Reflectivity of room surface $(\rho)$ & Wall Ceiling, Floor & 0.6 \\
\hline Transmitter Parameters $\left(\mathrm{T}_{\mathrm{x}}\right)$ & Pattern Mode & 1 or 2 \\
\hline
\end{tabular}




\begin{tabular}{|l|c|c|}
\hline & $\mathrm{X}$ & 3 \\
& $\mathrm{Y}$ & 2 \\
& $\mathrm{Z}$ & 3 \\
\hline Receiver Parameters. $\left(\mathrm{R}_{\mathrm{x}}\right)$ & Area & $1 \mathrm{~cm}^{2}$ \\
& $\mathrm{FOV}$ & $80^{0}$ \\
& $\mathrm{X}$ & 3 \\
& $\mathrm{Y}$ & 2 \\
& $\mathrm{Z}$ & 1 \\
\hline Cut off frequency & $\mathrm{f}_{\mathrm{c}}$ & $3 \times 10^{3}$ \\
\hline Number of Input bits & $\mathrm{I} / \mathrm{P}$ & 10,000 or 20,000 \\
\hline Number of reflections & $\mathrm{K}$ & $1,2,3,4$ \\
\hline Thresholding in percentage $(\%)$ & Th $(\%)$ & $10 \%, 20 \% 30 \%, 40 \%, 50 \%$ \\
\hline
\end{tabular}

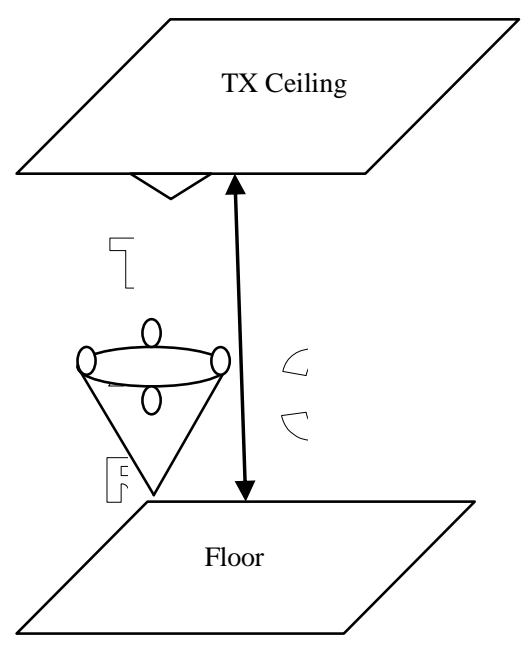

is of importance.

\section{i) Simulation Methodology for FFT Approach.}

The simulation analysis of Nondirected IR wireless channel was carried for the similar room configuration as in case of time domain analysis earlier. The simulation parameters used for this approach are listed in Table 1 as follows:

\section{ii) Results of FFT Analysis}

The performance analysis of Nondirected IR wireless channel using FFT has been discussed from the plots obtained in Figure 2 and Figure 3 below. For the simplicity we have displayed only the limited results.

\section{Modelling of Non Directed IR Channel Using FFT}

case of FFT analysis our attention was to obtain the percentage (\%) bit error rate (BER) change. Further the simulation was carried out by applying the thresholding of $10 \%, 20 \%, 30 \%$, $40 \%$ and $50 \%$. The BER was calculated by discarding the number of samples and considering the multipath reflections up to third order. The used.

The transmitter and receiver geometry in rectangular room is described in Fig.1. Since the channel modeling is done in an empty rectangular room, the geometry of the transmitter and receiver input samples of about 10000 and 20000 were 
Figure 2 and Figure 3 below. For the simplicity we have displayed only the limited results

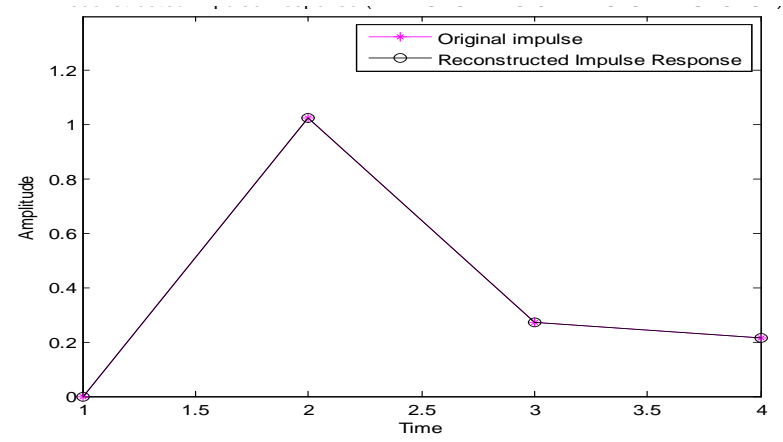

(a)

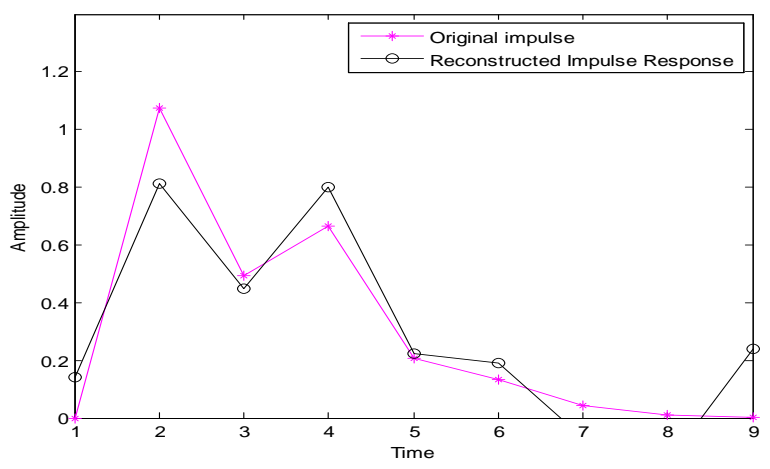

(b)

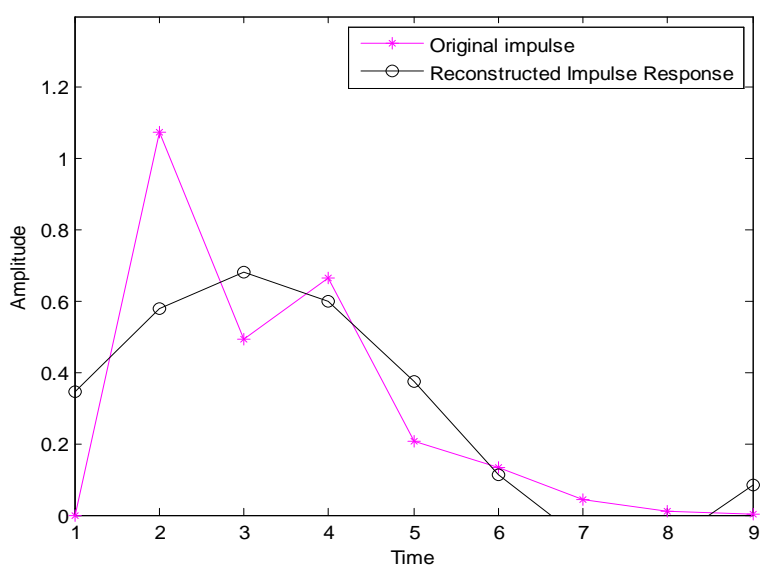

(c )

Fig.2.Original and reconstructed IR channel impulse responses for Thresholding of : (a) $10 \%$, (b) $20 \%$ and ( c ) $30 \%$ 


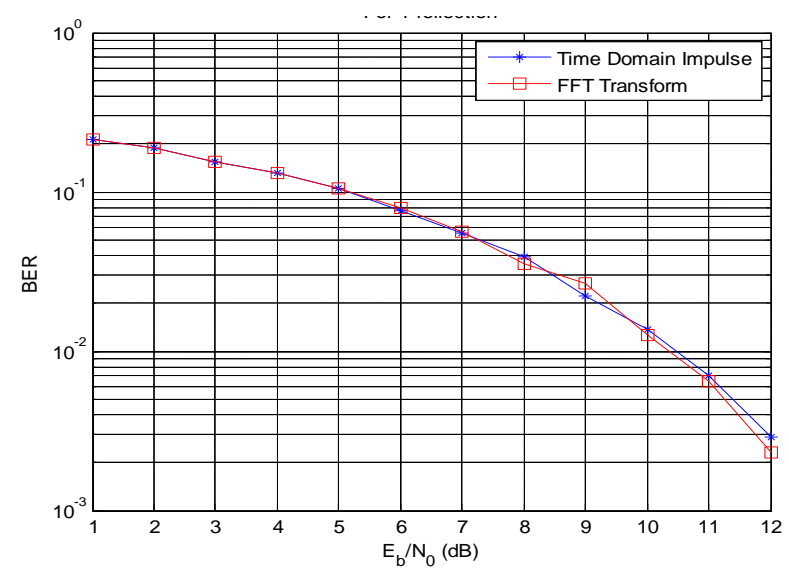

(a)

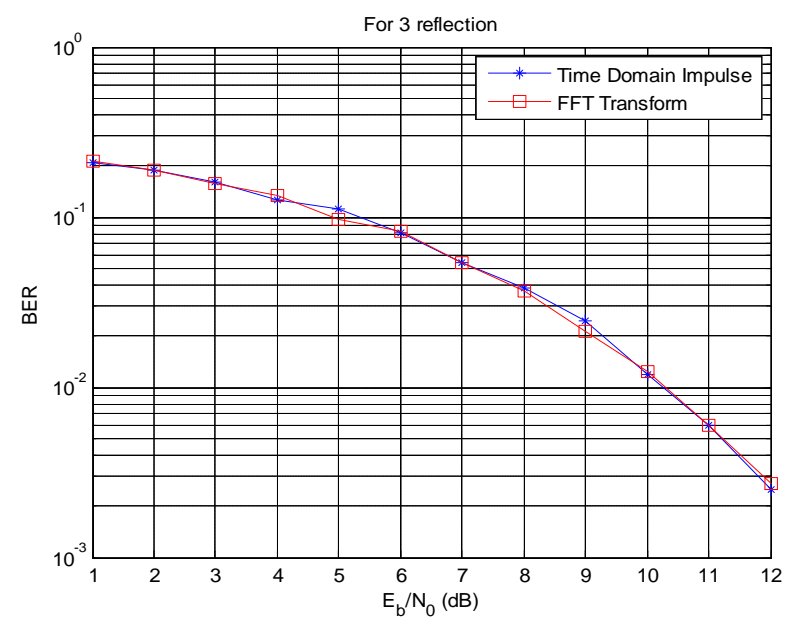

(b)

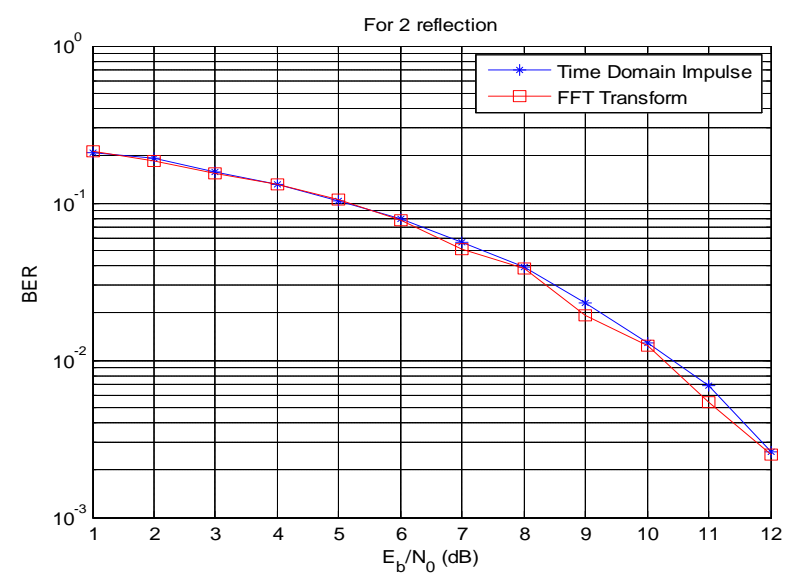

(c)

Fig. 3. $E_{b} / N_{o}$ Versus BER (a) $\mathrm{Th}=10 \%$,Reflection=1

(b) $\mathrm{Th}=20 \%$, Reflection $=2$ (c) $\mathrm{Th}=30 \%$, Reflection $=3$ 
TABLE 2: PERCENTAGE BER CHANGE AND DISCARDED COEFFICIENTS FOR FOUR REFLECTIONS

\begin{tabular}{|c|c|c|c|c|c|c|c|c|c|c|c|c|}
\hline \multirow{3}{*}{ 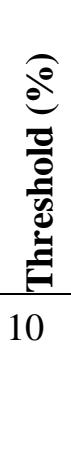 } & \multicolumn{3}{|c|}{ For 1 Reflection } & \multicolumn{3}{|c|}{ For 2 Reflection } & \multicolumn{3}{|c|}{ For 3 Reflection } & \multicolumn{3}{|c|}{ For 4 Reflection } \\
\hline & $\begin{array}{l}\% \\
\text { chang } \\
\text { Min }\end{array}$ & $\begin{array}{r}\text { BER } \\
\text { e Max, }\end{array}$ & $\begin{array}{l}\text { No. } \\
\text { Discarde } \\
\text { d out of } \\
1359 \\
\end{array}$ & $\begin{array}{l}\% \\
\text { chang } \\
\text { Max, } \\
\text { Min }\end{array}$ & $\mathrm{e}^{\text {BER }}$ & $\begin{array}{l}\text { No. } \\
\text { Discarde } \\
\text { d out of } \\
2456\end{array}$ & $\begin{array}{l}\% \\
\text { chang } \\
\text { Max, } \\
\text { Min }\end{array}$ & $e^{\text {BER }}$ & $\begin{array}{l}\text { No. } \\
\text { Discarde } \\
\text { d out of } \\
3362\end{array}$ & $\begin{array}{l}\% \\
\text { chan } \\
\text { Max, } \\
\text { Min }\end{array}$ & ge & $\begin{array}{l}\text { No. } \\
\text { Discarde } \\
\text { d out of } \\
4463\end{array}$ \\
\hline & $\begin{array}{l}- \\
0.037\end{array}$ & $\begin{array}{l}- \\
0.129 \\
6\end{array}$ & 126 & $\begin{array}{l}0.022 \\
0\end{array}$ & $\begin{array}{l}- \\
0.31 \\
6\end{array}$ & 466 & $\begin{array}{l}0.152 \\
5\end{array}$ & $\begin{array}{l}- \\
0.14 \\
4\end{array}$ & 815 & $\begin{array}{l}0.02 \\
7\end{array}$ & $\begin{array}{l}- \\
0.333 \\
3\end{array}$ & 1170 \\
\hline 20 & $\begin{array}{l}- \\
0.059\end{array}$ & $\begin{array}{l}- \\
.2273\end{array}$ & 440 & $\begin{array}{l}0.127 \\
6\end{array}$ & $\begin{array}{l}- \\
0.12 \\
9\end{array}$ & 1411 & $\begin{array}{l}0.027 \\
4\end{array}$ & \begin{tabular}{|l}
- \\
0.18 \\
1
\end{tabular} & 2269 & $\begin{array}{l}0.25 \\
5\end{array}$ & $\begin{array}{l}- \\
0.711 \\
1\end{array}$ & 3206 \\
\hline 30 & $\begin{array}{l}- \\
0.493\end{array}$ & $\begin{array}{l}- \\
92.05 \\
4\end{array}$ & 780 & $\begin{array}{l}1.350 \\
5\end{array}$ & $\begin{array}{l}- \\
39.6 \\
5\end{array}$ & 2119 & $\begin{array}{l}- \\
0.793\end{array}$ & \begin{tabular}{|l}
- \\
92.9 \\
3
\end{tabular} & 3115 & \begin{tabular}{|l}
- \\
0.88 \\
5
\end{tabular} & $\begin{array}{l}- \\
03.42 \\
0\end{array}$ & 4266 \\
\hline 40 & -0.70 & $\begin{array}{l}- \\
136.9 \\
2\end{array}$ & 1104 & $\begin{array}{l}38.36 \\
1\end{array}$ & $\begin{array}{l}- \\
237 . \\
9\end{array}$ & 2401 & $\begin{array}{l}37.06 \\
4\end{array}$ & $\begin{array}{l}- \\
230 . \\
7\end{array}$ & 3351 & $\begin{array}{l}- \\
1.35 \\
4\end{array}$ & $\begin{array}{l}- \\
59.69 \\
4\end{array}$ & 4456 \\
\hline 50 & \begin{tabular}{|l}
- \\
1.247 \\
8
\end{tabular} & $\begin{array}{l}- \\
175.1 \\
4\end{array}$ & 1274 & $\begin{array}{l}- \\
1.345 \\
6\end{array}$ & $\begin{array}{l}- \\
237 . \\
3\end{array}$ & 2453 & $\begin{array}{l}- \\
1.358\end{array}$ & $\begin{array}{l}- \\
249 . \\
2\end{array}$ & 3359 & \begin{tabular}{|l}
- \\
1.35 \\
4
\end{tabular} & $\begin{array}{l}- \\
165.7 \\
6\end{array}$ & 4460 \\
\hline
\end{tabular}

\begin{tabular}{|c|c|c|c|}
\hline SNR/BER & $\begin{array}{l}\text { TH=10 } \\
\text { \%, } \\
\text { REF=1 }\end{array}$ & $\begin{array}{c}\text { TH=30 } \\
\text { \%, } \\
\text { REF=2 }\end{array}$ & $\begin{array}{l}\text { TH=50 \%, } \\
\text { REF=3 }\end{array}$ \\
\hline 1 & 0.0127 & 0.0165 & 0.0202 \\
\hline 2 & -0.0267 & 0.0292 & $0.0077 \quad 0$ \\
\hline 3 & -0.006 & -0.0048 & -0.0382 \\
\hline 4 & 0.0305 & 0.0176 & -0.0449 \\
\hline 5 & -0.0657 & -0.0377 & -0.0101 \\
\hline 6 & -0.0657 & 0.0286 & -0.0265 \\
\hline 7 & -0.0941 & .0125 & -0.0272 \\
\hline 8 & -0.0339 & -0.0335 & 0.0721 \\
\hline 9 & -0.0978 & -0.1787 & -0.1111 \\
\hline
\end{tabular}




\begin{tabular}{|c|c|c|c|}
\hline 10 & 0.0189 & 0.081 & -0.05 \\
\hline 11 & 0.041 & 0.0156 & -0.3143 \\
\hline 12 & -0.4884 & 0.0469 & -0.120 \\
\hline
\end{tabular}

TABLE 3:SNR VERSUS BER FOR THRESHOLDING $10 \%, 30 \%, 50 \%$ AND REFLECTIONS 1,2 AND 3

In this FFT model considering 1, 2, 3 and 4 multiple bounce reflections were used to compare the 8-PPM IM/DD Non directed IR link with that of time domain IR Link channel model. We have computed the percentage in BER change for the number of reflections and percentage of Fourier coefficients discarded.

\section{Percentage Change In Bit Error Rate Estimation}

Our major interest was to estimate the change in percentage bit error rate (BER) of the impulse response obtained after applying the FFT and the impulse response obtained after applying the Inverse FFT (IFFT) It also estimated the number of the discarded coefficients by varying the thresholding and number of reflections. Also, the value of signal-to-noise-ratio (SNR) was changed from 1 to 12 in order to estimate the bit error rate (BER) for certain thresholding and reflection order. Table 2 has provided the values maximum and minimum percentage change in BER and number of discarded coefficients for $10 \%$ to $50 \%$ thresholding and 1 to 4 reflections.

To estimate the bit error rate (BER) for certain thresholding and reflection order. Table 2 has provided the values maximum and minimum percentage change in BER and number of discarded coefficients for $10 \%$ to $50 \%$ thresholding and 1 to 4 reflections.

\section{Discussion on FFT Results}

From the results obtained in Fig. 2 and Fig. 3, it is observed that the IR channel model in transform domain using FFT is analyzed using 8-PPM IM/DD IR wireless communication system. In case of FFT Model, we have applied the thresholding technique to discard the Fourier coefficients when implemented for the performance evaluation. The Table 2 lists the outcome of the simulation experiment.

In this FFT model considering 1, 2, 3 and 4 multiple bounce reflections were used to compare the 8-PPM IM/DD Non directed IR link with that of time domain IR Link channel model. We have computed the percentage in BER change for the number of reflections and percentage of Fourier coefficients discarded.

\section{IR Channel Analysis Using Wavelet}

We analyzed the IR channel by using Wavelet packet domain. In this section we carried out the performance evaluation of Nondirected IR channel for linear time variant and linear time invariant 
communication system in indoor environment.. The IR channel performance was also evaluated for different wavelets such as Db1, Haar, Sym2, Coif 2 etc.

The simulation parameters used for analysis of IR wireless channel using wavelet are same as used in FFT.

We used three level binary tree compositions during the simulation process. For the simulation we have taken into account two types of IR channels, namely time variant and time invariant channels. Figure 4 and 5 (a) and (b) shows the plot of percentage of wavelet packet coefficients kept versus mean square error and original and reconstructed IR channel.

i) Simulation Results using Wavelet:
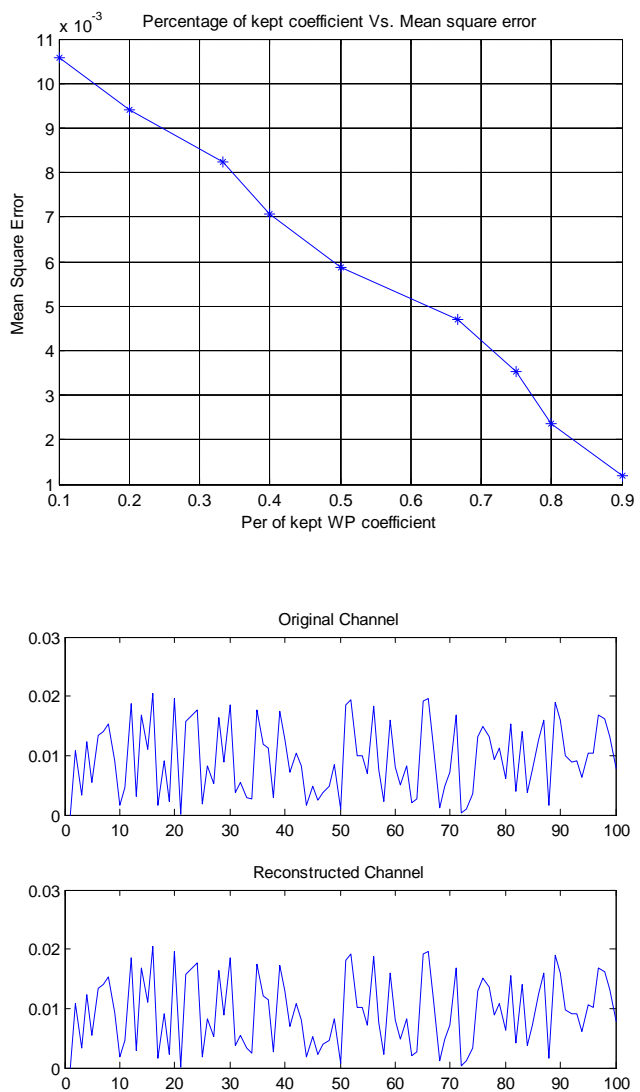

Figure 4 (a) Mean square error vs. \% of kept coefficient.

(b) Original and reconstructed IR Wireless channel. 

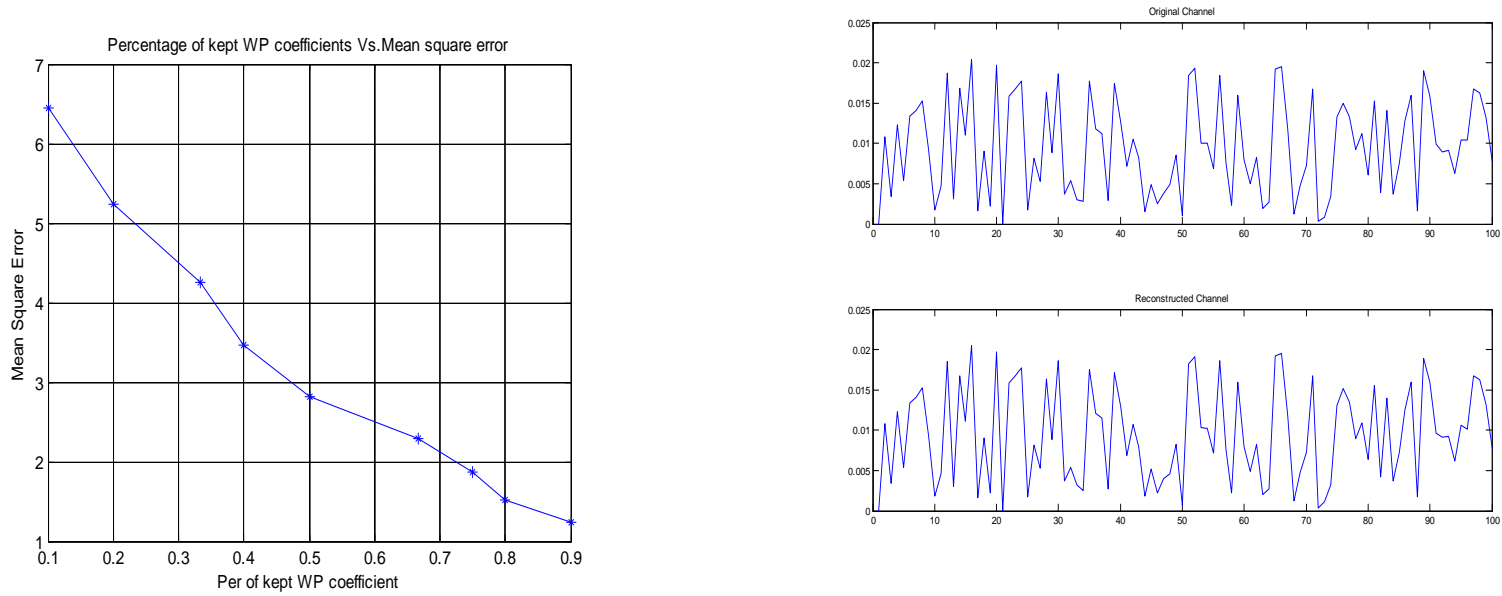

Figure 5 (a) Mean square error Vs. \% of kept coefficient. (b) Original and reconstructed IR time invariant channel

iii )

Discussion on Results

We first applied WP-based IR channel model for the time invariant channel. In the reconstruction, we kept only the most significant $60 \%$ of the WP coefficients and the channel is well. Here, it is observed that original and reconstructed impulse response is almost identical for both time variant and time invariant IR channel.

\section{Conclusion}

It was concluded that the performance by employing four (4) reflections model with $20 \%$ Fourier coefficients discarded was equivalent to the time domain model.

The major contribution of this research is that only 2890 of the transform domain coefficients are sufficient to model the Nondirected IR wireless channel which is equivalent to the time domain model. From the SNR values it is observed that as the SNR value increases as BER decreases.

The values of percentage bit error rate change for reflections, 1 to 4 and thresholding varying from are tabulated in TABLE 2. given below. Also, the BER values for different threresholding values $(10 \%, 30$ $\%$ and $50 \%$ ) and multiple reflections (Ref 1, Ref 2 and Ref 3 ) are stated in TABLE 3.

From the above results it was observed that as the percentage of WP coefficients increased the mean square error decreased. In linear time variant IR channel, the maximum and minimum values of the mean square error (MSE) to be observed were 0.0095 and 0.0011 respectively. Whereas in linear time invariant IR channels the maximum and minimum values of mean square error (MSE) to be observed were 1.8636 and 0.2071 respectively. Thus the mean square error in time variant IR channel was lower in comparison with that for linear time invariant IR channel. 


\section{Acknowledgment}

Dr. Prakash Patil thanks Hon. Dr. Vijay D. Patil President D. Y. Patil College of Engineering Ambi, Pune, Dr. Abhay A. Pawar, Principal and Mr Bhavanrao S. Gaikwad, Administrative officer for helping and supporting me to empower my research work

\section{References}

[1] R Pandya, "Emerging mobile and personal communication. System," IEEE Commun. Mag., vol.33, no. 6, pp.44-52, 1995.

[2] R.O. LaMaire, A. Krishna, P. Bhagwat and J. Panian, "Wireless LAN and mobile networking; standards and future directions" IEEE Communication. Mag., vol. 34, no. 8, pp. 86-94, 1996.

[3] K. Pahalvan, A. Zahedi and P. Krishnamurthy, "Wideband local access wireless LAN and wireless ATM," IEEE communication.

[4] A. G. Bell, "On the production and reproduction of sound by light", American Journal of Science, vol. XX, no. 118,pp. 305-324, Oct.1880

[5] F. R. Gefller and U. H. Bapst, "Wireless in-house data

Communication via diffuse infrared radiation," Proc. IEEE,D. Hash, J. Hillery, and J. White, "IR roomnet: Model and vol. 67, pp.1474-1486, Nov. 1979. Measurement,"IBM Commun. ITL Conf., June 1986.

[6] D. Hash, J. Hillery, and J. White, "IR roomnet: Model and vol. 67, pp.1474-1486, Nov. 1979. Measurement,'IBM Commun. ITL Conf., June 1986

[7] P.Hortensius, "Research and development plan of the infrared portable data link," internal report, IBM T. J. Watson Res. Cent., Yorktown Heights New York, 1990

[8] Joseph M. Kahn, W. J. Krause et.al, "Experimental Characterization of Nondirected indoor infrared channels", IEEE Trans.on communication, Vol. 43, No. 2/3/4,Feb/Mar/April 1995, pp 1613 - 623.

[9] J.R. Barry, "Wireless infrared communication, "Boston. Mass, London, Kluwer Academic, 1994.

[10] John R. Barry, Joseph M. Kahn et.al, "Simulation of Multipath Impulse Response for Indoor Wireless Optical Channels", IEEE Jr. on selected areas in communication, Vol. 11, No. 3, April 1993, pp $367-38$.

[11] R. Polikar , “The engineer's ultimate guide to wavelet analysis: the WaveletTutorial,"2005, http:// Users.rowan.edu/ polikar/WAVELET/WTutorial html.

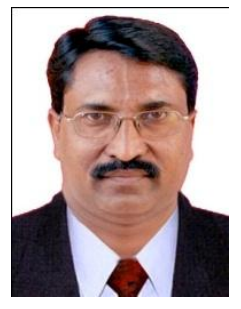

Dr.Prakash H. Patil is presently working as Vice Principal, Professor, HOD (E\&TC) at D Y Patil college of Engineering, Ambi, Pune. He has completed B.E. (Electronics), M.E. (Electronics) from Shivaji University Kolhapur and $\mathrm{PhD}$ (Electronics) from RTM's 
Nagpur University, Nagpur. He has total experience of 26 years in academics/Industry (R\&D). He is having 67 plus research publications in International/National Journals, conferences to his credit. His research area includes Mobile communication, Optical fiber communication, Wireless Networks, Optical Networks etc. at UG/PG level.

He is Fellow member of IETE and Life member of ISTE. He is Vice Chairman of IETE Pune, India. He is TPC member of NJIR, many IEEE conferences, International journals. He has received the prestigious MORAYA PURASKAR 2015 for his outstanding contribution in Technical education, spiritual work and social work. 